\title{
Delayed Sleep Phase Disorder in Children With Non- Monosymptomatic Primary Nocturnal Enuresis
}

\author{
Parsa Yousefichaijan ${ }^{\mathrm{a}}$, Ali Khosrobeigi ${ }^{\mathrm{b}}$, , Bahman Salehic, Hassan Taherahmadi ${ }^{\mathrm{a}}$,

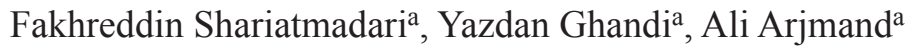

\begin{abstract}
Background: Delayed sleep phase disorder (DSPD), a circadian rhythm disorder, involves a significant, persistent, and intractable phase shift in sleep-wake schedule (later sleep onset and wake time) that conflicts with the individual's normal school, work, and/or lifestyle demands. Nocturnal enuresis (NE) is one of the most frequent pediatric pathologies. The prevalence of primary nocturnal enuresis (PNE) is around $9 \%$ in children aged 5 - 10 years and about $40 \%$ of them have one or more episodes per week.
\end{abstract}

Methods: In this study, we recruited 160 children with monosymptomatic primary nocturnal enuresis (MPNE) and 160 healthy children without MPNE aged 6 - 18 years old. The children sleep pattern was completed by the parents. Data were analyzed using $t$-test and Chisquare tests.

Results: Among 320 children in both groups, DSPD was significantly different between cases and controls.

Conclusions: Considering the results of this study, the higher prevalence of DSPD in children with non-monosymptomatic primary nocturnal enuresis (NMPNE) highlights the importance of early intervention for better treatment and prevention of DSPD in children.

Keywords: Delayed sleep phase disorder; Nocturnal enuresis; Child

\section{Introduction}

Delayed sleep phase disorder (DSPD) may occur at any age,

Manuscript accepted for publication June 29, 2015

${ }^{a}$ Department of Pediatrics, Arak University of Medical Sciences, Arak, Iran bDepartment of Student Research Committee, Arak University of Medical Sciences, Arak, Iran

'Department of Psychiatry, Arak University of Medical Sciences, Arak, Iran ${ }^{\mathrm{d} C o r r e s p o n d i n g ~ A u t h o r: ~ A l i ~ K h o s r o b e i g i, ~ D e p a r t m e n t ~ o f ~ S t u d e n t s ~ R e s e a r c h ~}$ Committee, Arak University of Medical Sciences, Arak, Iran.

Email: ali.khosrobeigi@arakmu.ac.ir

doi: http://dx.doi.org/10.14740/ijcp210w but is most common in adolescents and young adults. Individuals with DSPD often start out as night owls, that is, they have an underlying predisposition or circadian preference for staying up late at night and sleeping late in the morning, especially on weekends, holidays, and summer vacations. The underlying pathophysiology of DSPD is still unknown, although some authors have theorized that it involves an intrinsic abnormality in the circadian oscillators that govern the timing of the sleep period. Studies indicate that DSPD affects approximately $7-16 \%$ of adolescents. The most common clinical presentation is sleep initiation insomnia when the individual attempts to fall asleep at a "socially acceptable" desired bedtime, accompanied by extreme difficulty getting up in the morning even for desired activities, and daytime sleepiness. Sleep maintenance is generally not problematic, and no sleep onset insomnia is experienced if bedtime coincides with the preferred sleep onset time. School tardiness and frequent absenteeism are often present. Enuresis is defined as the repeated voiding of urine into clothes or bed at least twice a week for at least 3 consecutive months in a child who is at least 5 years of age [1-3]. The behavior is not due exclusively to the direct physiologic effect of a substance or a general medical condition [4,5]. Diurnal enuresis defines wetting while awake and nocturnal enuresis (NE) refers to voiding during sleep $[6,7]$. Primary enuresis occurs in children who have never been consistently dry through the night, whereas secondary enuresis refers to the resumption of wetting after at least 6 months of dryness [8]. Monosymptomatic enuresis has no associated daytime symptoms, and nonmonosymptomatic enuresis, which is more common, often has at least one subtle daytime symptom [9].

\section{Materials and Methods}

To perform this case-control study, we assessed the prevalence of DSPD in 160 children aged 6 - 18 years with monosymptomatic primary nocturnal enuresis (MPNE) and compared the results with a control group that included 160 healthy children with the same age, sex, socioeconomic status, level of education and relationship between parents. In this case-control study, we selected 160 children with non-monosymptomatic primary nocturnal enuresis (NMPNE) based on the DSM IV criteria [2] as cases and 146 children without NMPNE as the controls who were all admitted to Amir-Kabir Hospital, Arak, 
Iran. Our exclusion criteria were: 1) children with known underlying kidney diseases or genitourinary problems; 2) children with psychological disorders and/or mental retardation or nervous system disorders; 3 ) children whose parents did not cooperate fully, e.g. did not fill the forms completely or decided to withdraw from the study. The control group was selected from pediatric patients with other complaints like trauma, stomachache, back pain, and common cold considering the exclusion criteria. This questionnaire was tested for reliability in a pilot study by researchers with 50 patients in each of the case and control groups. The results were analyzed with SPSS 19 using descriptive statistics for basic information. P values less than 0.05 were considered significant in our comparisons. This study was confirmed by the Ethics Committee of Arak University of Medical Sciences.

\section{Results}

Overall, 160 cases and 160 controls were selected for study. Demographic data showed that $53.4 \%$ of the participants were boys and $46.6 \%$ were girls. Moreover, the mean age of children was $7.8 \pm 1.1$. In the control group, $41.8 \%$ were boys and $58.2 \%$ were girls with a mean age of $7.7 \pm 1.4$. Our results showed a significant difference in some of the categories.

\section{Discussion}

In this study, we compared behavioral problems in children with and without NMPNE. Based on the results, mental problems and depression were the most frequent while decreased attention, hyperactivity, and physical problems had the lowest frequency in both case and control groups. Moreover, the data revealed that the mean score of some behavioral problems in NMPNE children was three to four times more than healthy children. Compared with the sleep of control subjects, the natural sleep of children with NE is significantly more fragmented, and the children with NE experience higher levels of daytime sleepiness. This phenomenology is associated with bedwetting episodes and attempts to keep the child dry during the night. These findings may suggest that children with NE suffer from sleep fragmentation, which may explain their higher arousal threshold. These findings have clinical implications for enuresis management [10]. Gloria demonstrated in this unselected group of children with sickle cell anemia, sleep disordered breathing (SDB) was associated with enuresis. Results of this study support that children with sickle cell anemia who present with enuresis should be evaluated by a pulmonologist for SDB [11]. Kushnir et al suggested that sleep patterns of school-aged children with enuresis who do not wear night diapers are impaired, and the sleep quality of children using night diapers is similar to those of healthy children. Thus, clinicians and healthcare providers should consider recommending sleeping with night diapers for untreated children with enuresis, based on its positive impact on sleep [12]. Snoring in children with monosymptomatic NE puts them at increased risk for behavioral and psychosocial problems, in addition to impaired health-related quality of life. These findings support the need for future studies of the neurological links between SDB and monosymptomatic NE [13]. Bascom et al in study confirms the link between SDB and enuresis. All pediatric health care providers should be aware of this risk. The risk may be magnified in patients with concomitant daytime incontinence [14]. Brooks concluded that there is a high prevalence of enuresis in children with suspected SDB. This may be due to the effects of obstructive sleep apnea on arousal response, bladder pressure, or urinary hormone secretion [15]. SDB in children is associated with NE. Adenotonsillectomy is associated with a significant improvement in enuresis in children with SDB. There is a need for randomized controlled trials to look at the role adenotonsillectomy in children with SDB and enuresis [16].

\section{Conclusion}

Our study results showed a higher incidence of DSPD in children with NMPNE. Also, based on the results of other studies, treatment of DSPD reduces the problem of primary nocturnal enuresis (PNE). It seems that timely diagnosis and treatment can prevent the continuation of this disorder and the development of other sleep disorders. We suggest that children with PNE should be evaluated for other sleep disorders in an attempt to resolve the problem.

\section{Acknowledgement}

This paper was the result of a student thesis. We would like to express our gratitude to all the people who supported and assisted us.

\section{References}

1. Yousefichaijan P, Salehi B, Rafiei M, Firouzifar MR, Mousavinejad SA. Parents function and behavioral disorders in children with/ without diurnal voiding dysfunction: A comparative study. ZJRMS. 2013;15.

2. Yousefichaijan P, Soltani P, Haghverdi F, Saeedi N, Salehi B, Sharafkhah M, Taherahmadi H. Parents function and behavioral disorders in children with and without renal transplant recipient's: A comparative study. Experimental and Clinical Transplantation. 2014;12(supplement 2):180.

3. Yousefichaijan P, Salehi B, Firouzifar MR, Sheikholeslami H. The correlation between attention deficit hyperactivity disorder and enuresis in children with nocturnal enuresis. JIMS. 2012; 30(184 2nd week):1-8.

4. Yousefichaijan P, Firouzifar MR, Cyrus A. Correlation between sacral ratio and primary enuresis. J Nephropathology. 2012;1(3):183-187.

5. Yousefichaijan P, Firouzifar MR, Dorreh F. Growth and development in 6 years old children with and without primary nocturnal enuresis. Journal of Medical University of Zanjan 2011;20(82):97-100. 
6. Yousefichaijan P, Salehi B, Rafiei M, Ghadimi N, Taherahmadi H, Hashemi SM, Naziri M. Emotional Disorders in Children with Monosymptomatic Primary Nocturnal Enuresis. J Ped Nephrology. 2015;3(1):22-25.

7. Yousefichaijan P, Salehi B, Rafiei M, Taherahmadi H, Sharafkhah M, Naziri M. Prevalence of ObsessiveCompulsive Disorder in Pediatric and adolescent Patients with Chronic Kidney Disease. J Ped Nephrology. 2014;2(3):107-109.

8. Yousefichaijan P, Khosrobeigi A, Zargar S, Salehi B. Sleep Disorder in Children With Overactive Bladder. Int J Clin Pediatr. 2015;4(2-3):145-148.

9. Yousefichaijan P, Salehi B, Khosrobeigi A, Hajirahimi M, Rafiei M, Taherahmadi M. Prevalence of obsessive-compulsive disorder in pediatric patients with the daytime frequency syndrome of childhood or pollakiuria. Arak Medical University Journal. 2014:17(89):80-87.

10. Cohen-Zrubavel V, Kushnir B, Kushnir J, Sadeh A. Sleep and sleepiness in children with nocturnal enuresis. Sleep. 2011;34(2):191-194.

11. Lehmann GC, Bell TR, Kirkham FJ, Gavlak JC, Ferguson
TF, Strunk RC, Austin P, et al. Enuresis associated with sleep disordered breathing in children with sickle cell anemia. J Urol. 2012;188(4 Suppl):1572-1576.

12. Kushnir J, Cohen-Zrubavel V, Kushnir B. Night diapers use and sleep in children with enuresis. Sleep Med. 2013;14(10):1013-1016.

13. Wolfe-Christensen C, Kovacevic LG, Mirkovic J, Lakshmanan Y. Lower health related quality of life and psychosocial difficulties in children with monosymptomatic nocturnal enuresis--is snoring a marker of severity? J Urol. 2013;190(4 Suppl):1501-1504.

14. Bascom A, Penney T, Metcalfe M, Knox A, Witmans M, Uweira T, Metcalfe PD. High risk of sleep disordered breathing in the enuresis population. J Urol. 2011;186(4 Suppl):1710-1713.

15. Brooks LJ, Topol HI. Enuresis in children with sleep apnea. J Pediatr. 2003;142(5):515-518.

16. Jeyakumar A, Rahman SI, Armbrecht ES, Mitchell R. The association between sleep-disordered breathing and enuresis in children. Laryngoscope. 2012;122(8):18731877. 